\author{
Military Technical College \\ Kobry El-Kobbah, \\ Cairo, Egypt.
}

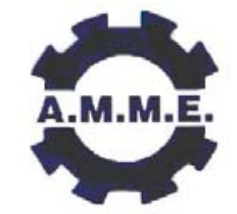

\title{
MATERIAL PROPERTY MEASUREMENTS USING NDT METHODS
}

BALASUBRAMANIAM* $\mathrm{K}$.

\begin{abstract}
In this paper, a summary of some of the NDT methods that can be used for the evaluation of material properties such as stiffness, strength, residual stress, plastic deformation, creep damage, fatigue damage, etc. will be addressed. NDT methods includes ultrasonics, electromagnetics, and thermal. In some applications, the NDT methods provides a direct measurement of the required property, while in others, the correlation is indirect.
\end{abstract}

\footnotetext{
* Center for Nondestructive Evaluation and Department of Mechanical Engineering, Indian Institute of Technology Madras, Chennai INDIA 600036. E-mail: balas@iitm.ac.in
} 


\section{INTRODUCTION}

Nondestructive methods for Materials Characterization can be defined as "NDE methods for determination of microstructure and distributed properties for structural materials". NDE methods have the potential for making mechanical, physical, and chemical measurements both in laboratory as well as in the field. These techniques are being used in the characterization of a wide range of materials, from traditional materials such as metals and wood to new materials such as composites and electronic wafers. Material characterization measurements are required in every facet of a product life cycle, i.e. from the materials development and design to manufacturing to failure analysis.

Any NDE experiment, when modeled in terms of systems approach, involves three ingredients:

1. The input signal provided to the material/structure in the form of ultrasonics, heat, eddy current, etc.

2. The Material or Structure itself that interacts and modifies the signal, and

3. The output signal that is recorded by a receiver transduction system.

This is illustrated in Fig. 1. During the theoretical modeling, it is customary to assume that the necessary information regarding the input signal and the Material/Structures (geometry, properties, boundary conditions, etc.) are known. Based on this assumption models can be developed that attempts to predict the Output Signal. Such models can be categorized as Forward Models.

Hence, the Inverse Models represents efforts to exactly reverse this effort, i.e. assume that the output signal is provided and predict either the Input Signal or the Material Properties or in some cased both [1,2]. Often, in addition to the output signals, it is necessary to have additional information about either the input signal or the material properties such that only one set of unknowns can be evaluated.

While the forward models serve as useful tools in NDE for optimization of experimental parameters as well as assisting output signal interpretation, the inverse models are more useful during NDT inspection since the inspector has both the input and the output signal information and is in need for information on the state of the material that is being evaluated/measured.

\section{STIFFNESS DETERMINATION}

Elastic constants determination, particularly for anisotropic metals such as Centrifugally Cast Stainless Steel (CCSS) with columnar grain structure or rolled materials, is critical in the prediction of the performance of the structures manufactured using these materials. Ultrasonic data have been used in the material property measurements, specifically the elastic constant matrix, using inversion methods. Both Genetic Algorithms [3] as well as Artificial Neural Network [4] Methods have been shown to solve the inverse problem of measurement of elastic moduli from ultrasonic guided wave dispersion data sets. Similar methods have been employed for the use of velocity of ultrasound in specific planes for the reconstruction of elastic constants [5]. 


\section{PLASTIC DEFORMATION CHARACTERISATION}

Plastic deformation of materials are difficult to quantify, but reduces the remaining life of a material. The evaluation of the metal plastic deformation can be measured, quantitatively, using Nonlinear Ultrasonic, Thermal Imaging or Eddy-current based NDT methods.

\section{Nonlinear Ultrasonic Methods}

In the nonlinear ultrasonic harmonic generation technique, the material is characterized by magnitude of the harmonics generated $\left(A_{2} / A_{1}{ }^{2}\right)$, when a monochromatic finite amplitude sinusoidal signal passed through it. This technique involves the measurement of relative strengths of harmonics produced when a longitudinal or Raleigh wave propagates through the material under study. Non-linear ultrasonic technique has been used to study the effect of plastic deformation in an aluminum alloy by Hikata et al [6]. Scorey [7] has reported the variation of nonlinear parameter $(\beta)$ under gradual unloading condition and found that the second harmonic is lower than its initial value, which recovered to its initial value over a period of time. The rate of recovery of the harmonic to the initial value was found to increase with temperature. Jhang and Kim [8] studied the variation of nonlinear parameter in plastically deformed stainless steel alloys using longitudinal mode and observed an increase in the nonlinear parameter as the applied stress increases. Very recently Hermann [9] observed significant changes in the nonlinearity in Raleigh waves in the plastically deformed specimens and as well in the longitudinal mode. A typical result obtained on Aluminum test samples is shown in Fig. 2 where it was observed that the nonlinear indicator $\mathrm{A}_{2} / \mathrm{A}_{1}{ }^{2}$ is nearly constant up to a certain level of residual plastic deformation and thereafter there is a significant increase in the non-linear parameter response [10].

\section{Thermal Imaging Method}

During Plastic Deformation, due to the strain energy released, there is a change in the surface temperature of the material that is now measurable using highly sensitive IR cameras. During mechanical loading several thermo-mechanical effects are activated. Thermoelasticity $\left(\Delta \mathrm{T}_{\mathrm{el}}\right.$ : temperature variation due to thermoelastic effects), heat dissipation by reversible anelastic damping, heat dissipation by irreversible plastic deformation $\left(\Delta \mathrm{T}_{\text {diss }}\right.$ : temperature variation due to heat dissipation), and surface friction (e.g. at internal surfaces) contribute to the observed change in temperature during loading. The temperature change that occurs during elastic loading $\left(\Delta \mathrm{T}_{\mathrm{el}}\right)$ varies in proportion to the applied stress $(\sigma)$ due to the Joule Thomson effect

$$
\Delta \mathrm{T}_{\mathrm{el}}=-(\alpha / \rho c) \mathrm{T}_{0} \Delta \sigma
$$

where, $\alpha$ is thermal expansion coefficient, density, $c$ specific heat, $T_{0}$ initial temperature.

\section{Eddy Current Methods}

Single frequency, multi frequency, and pulsed eddy current NDT methods can be used to measure the electrical conductivity and magnetic permeability of the material. It has been observed that the change in the conductivity of the material can be related to 
plastic deformation of the material. A typical result is shown in Fig. 3 where the amplitude of the pulse eddy current response is plotted as a function of strain of an aluminum alloy sample undergoing continuous loading up to failure. It can be observed that the slope of the response function shows a bi-linear behavior i.e. one for the elastic deformation and one for the plastic deformation, indicating the feasibility of the technique to quantitatively measure the plastic deformation in materials.

\section{FATIGUE DAMAGE MONITORING}

The evaluation of fatigue damage in materials be measured, quantitatively, using Nonlinear Ultrasonic and/or Thermal Imaging based NDT methods.

\section{Nonlinear Ultrasonic Methods}

Nonlinear ultrasonic harmonic generation measurements were conducted on the aluminum alloy AA7175-T7351 specimens fatigue cycled at the loading conditions described earlier. A sinusoidal pulse of frequency $2 \mathrm{MHz}$. is introduced in the specimen through an acrylic wedge and received at the other end using a $5 \mathrm{MHz}$. transducer. Six hourglass fatigue specimens were prepared for the fatigue damage studies, of which, a batch of three specimens were tested between $90 \%$ and $10 \%$ of yield strength. At periodic intervals of time (approximately $5 \%$ of fatigue life) the specimens were unloaded and tested for nonlinear ultrasonic response using surface acoustic wave mode (along the direction of applied load) and this procedure is continued till the specimen undergoes failure. As the fatigue damage initiates at the surface, surface acoustic wave was considered as appropriate technique compared to the longitudinal mode. Also the surface acoustic waves (Raleigh waves) have the following advantages; a) Raleigh waves can follow the surface around and this feature allows using in inspection of components with curved geometries. b) Raleigh wave's energy is concentrated near the free surface of a component, which can lead to stronger nonlinear effects compared to bulk waves, which is significant considering that the fatigue damage is a surface phenomenon; and (c) Raleigh waves propagate long distances without significant loss of energy. The magnitude of the nonlinear parameter varied from the top surface to bottom surface in different manner in different samples and this observation can help in predicting on which surface the crack can initiate first. As fatigue crack can initiate on either of the two surfaces of the specimen or in some cases in the mid-thickness region, it was considered appropriate to measure the NLU response on both the surfaces. It can be seen that the NLU response for the two surfaces show two distinct peaks for all the samples examined through the percentage of life at which they occur varies slightly. This variation is within 1000 cycles $(5 \%$ of fatigue life) and could be due to various factors which are within the scattering factor in the fatigue life. It was observed that the surface which showed a higher value of nonlinear parameter had the first crack initiation. From a typical result shown in Fig. 4, one can observe that the first one appears at about $40-50 \%$ fatigue life and the second peak at about $80-90 \%$. The occurrence of the second peak can be linked to appearance of a visible crack appears at one of the edges on the hourglass specimen. The NLU response thereafter drops within the next 500 cycles of fatigue loading. Visual inspection of the samples showed a small depression appearing near the edge of the specimen at which the crack appears and the crack was found to propagate along the width. It can also be observed from the figures that the magnitude of $A_{2} / A_{1}{ }^{2}$ varies from specimen to specimen. 


\section{Thermal Imaging Method}

During fatigue of a metal sample, a correlation between the temperature increase during short-term ultrasonic loading and accumulated fatigue cycles is used to suggest a methodology for in-field assessment of fatigue condition [12]. Early stages of damage are characterized by changes in the material sub-microstructure, which lead to crack initiation. It is well established in the open literature that heat dissipated during cyclic loading is sensitive to the fatigue state of a material [13-15]. The schematic representation of the thermal energy release during fatigure of a sample is illustrated in Fig. 5. It was shown that the dissipated heat in the tested material can be excited using high amplitude low frequency loading, or high power ultrasonic loading. Determination of the dissipated heat per loading cycle from short-term loading experiments allows the evaluation of microstructure variations due to fatigue independent of the thermal boundary conditions. A relationship between the thermal NDE parameter and the mechanical fatigue loading conditions was developed [12].

\section{CREEP DAMAGE MEASUREMENTS}

Ultrasonic measurements in the linear (velocity) and non-linear domains (second harmonic amplitude) to evaluate creep damage in a near- $\alpha$ IMI-834 titanium alloy, currently being used in the compressor module of aero-engines. The creep damage in this alloy has initiated in the form of microvoids at primary $\alpha /$ transformed $\beta$ interface and the volume fraction of voids increase progressively with creep deformation. Good agreement between the results and data obtained from metallographic studies indicated the usefulness of the method for in-service evaluation of creep damage. Non-linear ultrasonic technique was found to be significantly more sensitive, when compared to the linear ultrasonic velocity measurements, for the assessment of creep damage. Creep specimens of two different thicknesses i.e. 2 and $4 \mathrm{~mm}$ were fabricated from the heat treated specimen coupons by wire-cut electro discharge machining (EDM). Constant load creep tests were conducted in air at a temperature of $600^{\circ} \mathrm{C}$ and stress of $300 \mathrm{MPa}$. Extensometer was mounted on the specimens and two linear variable differential transducers (LVDTs) were attached to the extensometer outside the heat zone and the average of both transducers was used for plotting the creep curve. Creep test was carried out until fracture on a single specimen and based on the results/ curve of the test, another specimen was interrupted at different time interval for damage evaluation at different time interval. In the first series of the experiments, the consecutive variation of ultrasonic velocities with creep time was studied. The interrupted creep test was carried out for the four- specimens at different intervals. This cycle was repeated. The test was carried out under constant stress of $300 \mathrm{MPa}$ at $600^{\circ} \mathrm{C}$. For all creep tests, reference specimens were held in the furnace alongside the creep specimens. Reference specimens sustained no load. Measurements of both linear ultrasonic velocity and the non-linear parameter have been carried on interrupted creep tested specimens at a temperature of $873 \mathrm{~K}$ and under constant stress of 300 $\mathrm{MPa}$ and plotted in Figs. 7 and 8 . A $150 \%$ change in the non-linear parameter ' $\beta$ ' is observed as a function of creep fraction life as compared to $15 \%$ change in the linear velocity, which clearly proves that ' $\beta$ ' is much more sensitive than longitudinal velocity measurements. It can be observed that while there is a significant increase in the response to creep damage up to 72 hours, it was observed that the response drops on samples of 98 hours of creep damage. This behavior may be explained by the increase 
in the microstructure features such as voids with increasing exposure to creep damage. Since, the Non-linear ultrasonic response is a function of scale of the damage, there is an optimal size of the damage scale that provides the higher NLU response. Any further increase in the damage scale would cause a reduction in this response and have also been observed by Ogi et. al [16] for fatigue damage in steels.

\section{STRESS MEASUREMENTS}

The measurement of applied and residual stress state of the critical components is allthe-more vital for ensuring a safe performance of critical components and structures. There is an urgent need for methods that reliably measure the applied and residual stress in a wide range of components and material systems. The common methods of stress measurement that are used in the industry includes, but not limited to, X-ray Diffraction, Neutron diffraction, Magnetic Barkhausen Noise methods, and Ultrasonic methods. In this article, the use of ultrasonic methods for the measurement of stress will be described.

\section{Ultrasonic Methods}

Different wave modes of ultrasound may possess unique acousto-elastic constants and also may have specific advantages depending on the nature of the residual stress and the geometry and access available in the component that is being evaluated. The following wave modes are most commonly reported in the literature for the measurement of residual stresses.

$>$ Shear Wave Bi-refringence Method

$>$ Critically Refracted Waves (Lcr Waves)

$>$ Shear Horizontal Waves (SH waves)

$>$ Raleigh Waves (also called Surface waves)

$>$ Lamb Waves (also called plate waves)

The DEBRO 30 and DEBBIE instruments developed by Deputat [17.18], the set-up developed by the French Railroad SNCF by Limal [18] and the UER set-up developed by Herzer and Schneider [19] are the well known commercial systems that use the ultrasonic Lcr waves for stress measurement. The various ultrasonic methods for the measurement of residual stress has been summarized by Bray [20]

\section{Ultrasonic Raleigh Wave Dispersion Technique for Stress Profiling}

The residual stress in the peened components is not uniform with depth into the component. The stress is maximum, and compressive, at around 200 micron deep and then becomes tensile at around 300 microns. The conventional method of $x$-ray diffraction does not permit the evaluation of the maximum compressive residual stresses in such components since this method penetrates only less than 10 microns. Hence, a technique for the measurement of stress, as a function of depth up to 200-300 microns was found necessary. Broad-band ultrasonic guided Rayleigh wave modes were generated and received at the surface of the structure. The ultrasonic wave velocity profile, as a function of frequency, will be measured using phase spectrum method. The measured wave velocity profile will then be related to the stress gradient information using a novel inverse technique.[21] The short focal length (acoustic microscopy configuration) allows the transducer to simultaneously generate both 
longitudinal wave modes propagating perpendicular to the surface of the metallic structure and guided Raleigh waves in a tangential direction to the structure. The longitudinal wave mode reflection from the surface will be used to optimize the alignment of the transducer with the surface while the Raleigh wave will be used to measure the stress gradient. The technique exploits the dispersion phenomena of the ultrasonic surface hugging wave modes (Rayleigh waves). The residual stress gradients will directly influence the dispersion of the Rayleigh wave modes and is measured from the phase information in the ultrasonic signal. An inversion method based on Voltera integral inversion was employed to perform the multi-dimensional evaluation of stress gradient information from simulated and experimental data. Emphasis of this work limited to obtain data on stress measurements using the Raleigh wave dispersion technique and to demonstrate the "proof-of-concept" for metallic components made from aluminum alloys. The experimental results for 3 cases of different loads with decreasing loading are presented in Fig. 8. The phase difference .vs. the frequency data was used to invert the stress .vs. depth profile using the inversion algorithm developed here. It can be observed that the linear profile is observed. Also, all of the profiles intersect at a point very close to each other and also not very different from the zero stress level. It must be noted that the depth of the Raleigh wave that has penetrated the AL alloy sample is only about 2-3 $\mathrm{mm}$. Hence, the data that is plotted is an extrapolation of the measured data over the prescribed depth of the sample.

\section{Multi-Frequency Eddy Current for Stress Profiling}

This section describes a multi-frequency eddy current inversion procedure for characterizinglspecimens that are peened to improve fatigue strength. Multi-frequency inductance data was obtained by using well-characterized eddy current probes. The inversion uses a multi-layer axisymmetric finite element model as the forward model and the conductivity of each layer is found through interpolation of the inductanceconductivity data generated by the forward model. Skin depth approximation was used to isolate the integral effects of the conductivity variation on the inductance signal. Inverted conductivity profiles of the water jet peened specimens was found to resemble the predicted profiles. Information regarding the shape of residual stress gradients and relative intensities of peening were inferred from the conductivity profiles.[22]

Compressive (tensile) stresses cause an increase (decrease) in the electrical conductivity. Up to $5 \%$ increase in conductivity is obtained near the surface of waterpeened specimens. From the stress profiles of peened samples (Fig. 9), a stress reversal over depth was noted after peening wherein the mode changes from compressive in the top layer to a net tensile stress in the bulk of the material. Analogous to this effect, the conductivity of the specimen also changes its mode with respect to the substrate conductivity at depths of around 120 microns. It is further noted that the reversal depth increases when the peening pressures are increased. The multifrequency eddy current inversion technique is hence effective for assessing the near surface changes in conductivity due to peening and can be used to gauge information regarding the depth of reversal of residual stresses and relative intensities of peening between different samples. 


\section{SUMMARY}

In this paper, several NDT methods have been discuss as promising techniques for the quantitative measurement of materials state of metallic components and structures. The nondestructive nature of these methods makes it very attractive for application in the industry.

\section{REFERENCES}

[1] Roy D N G and Couchman L S, Inverse Problems and Inverse Scattering of Plane Waves, Academic Press, London.(2002).

[2] Liu, G.R.Inverse Methods in Nondestructive Evaluation, CRC Press, (2003)

[3] Balasubramaniam, K., and N. S. Rao, "Inversion of Composite Material Elastic Constants from Ultrasonic Bulk Wave Phase Velocity Data using Genetic Algorithms," Composites Part B : Engineering, 29(B), 171-180. (1998)

[4] Wheeler, E.S., R. King, and K. Balasubramaniam, "An Artificial Neural Network as a Tool for the Inversion of Ultrasonic Dispersion Data for Material Characterization," Review of Progress in Quantitative Nondestructive Evaluation, 18B, 1265-1272, (1999).

[5] Reddy, S.S.S. Krishnan Balasubramaniam, C.V.Krishnamurthy, and M. Shankar,," Ultrasonic goniometry immersion techniques for the measurement of elastic moduli,." Composite Structures, 67, 3-17, (2005)

[6] Hikata, A., B. B. Chick and C. Elbaum, (1965) Dislocation contribution to the second harmonic generation of ultrasonic waves. Journal of Applied Physics, 36 (1), 229.

[7] Scorey, C. R., (1970) Recovery of Ultrasonic Second-Harmonic Amplitude in an Aluminum Crystal. Journal of Applied Physics, 41, 2535.

[8] Kyung-Young Jhang, Kyung-Cho Kim, (1999) Evaluation of material degradation using nonlinear ultrasonics, Ultrasonics, 37, 39-44.

[9] Hermann J, MS thesis Georgia Institute of Technology, Atlanta, USA. 2005.

[10] Jayarao, V.V.S., M.S. Thesis, Indian Institute of Technology Madras, Chennai 600 036, India (2007).

[11] Rosner, H. S. Sathish, N. Meyendorf, in: Rev. of Prog. in QNDE, American Institute of Physics, 1702-20 1709 (2001).

[12] Meyendorf, N. H. Rosner, V. Kramb, S. Sathish,i Thermo-acoustic fatigue characterization Ultrasonics 40 427-434 (2002).

[13] Wong, A.K. G.C. Kirby, Eng. Frac. Mech. 37(3) 493-504 (1990).

[14] Jacobsen, T.K. B.F. Sorensen, P. Brondsted, Exper. Mech. 38(4) 289-294 (1998).

[15] Harwood, N. W.M. Cummings, in: Thermoelastic Stress Analysis, Adam Hilger, Bristol, 35-43 (1991)

[16] Ogi, H., M. Hirao, S. Aoki, Noncontact monitoring of surface wave nonlinearity for predicting the remaining life of fatigued steels. Journal of Applied Physics, 90(1), 438 (2001).

[17] Deputat, J., "Application of the acoustoelastic effect in measurements of residual stresses" Arch. Acoust. 15(1-2), 69-92,(1990).

[18] Deputat, J., Kwaszcynska-Klimek, A., Szelazek, J., "Monitoring of Residual Stress in Railroad Wheels with Ultrasound:, Proc. 12th World Congress, Elsevier Science Publishers B.V. Amsterdam, J.Boogaard, G.M. van Dijk (eds.) 974-976, (1989). 
[19] Limal, J.L., Del Fabro, V., "Prevention of Thermal Damage in Railroad Wheels thanks to the Monitoring of Residual Stresses by Ultrasonic Examination". Proc. 10th International Sympo-sium Assessment of Materials Aging and Damage Evolution by Non Destructive Evaluation Methods, TOME XVI, Cercle D'Etudes Des Metaux, St. Etienne Chapter 17, (1995).

[20] Herzer, R., Frotscher, H., Schillo, K.; Bruche, D., Schneider, E., "Ultrasonic SetUp to Characterize Stress States in Rims of Railroad Wheels" Nondestructive Characterization of Materials VI, Eds. R. E. Green Jr. et al, Plenum Press, New York 699-706, (1994).

[21] Bray, D. E., "Current Directions of Ultrasonic Stress Measurement techniques", 15th WCNDT, Roma (2000).

[22] Rajagopala, P., K. Balasubramaniam, S. Maddu, C.V. Krishnamurthy, "A new approach to inversion of surface wave dispersion relation for determination of depth distribution of non-uniform stresses in elastic materials", International Journal of Solids and Structures, 42, 789-803, (2004).

[23] Sundarajan, V. , K. Balasubramaniam, N Ramesh Babu, N Rajesh "A multifrequency eddy current inversion method for characterizing conductivity gradients on water jet peened components" NDT\&E, International 38 54-60 (2005) 


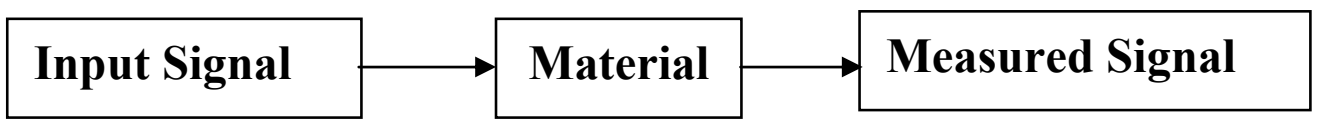

Fig.1: Typical NDT system in the forward sense.



Fig. 2 Variation of $A_{2} / A_{1}^{2}$ with plastic strain for Aluminum Samples

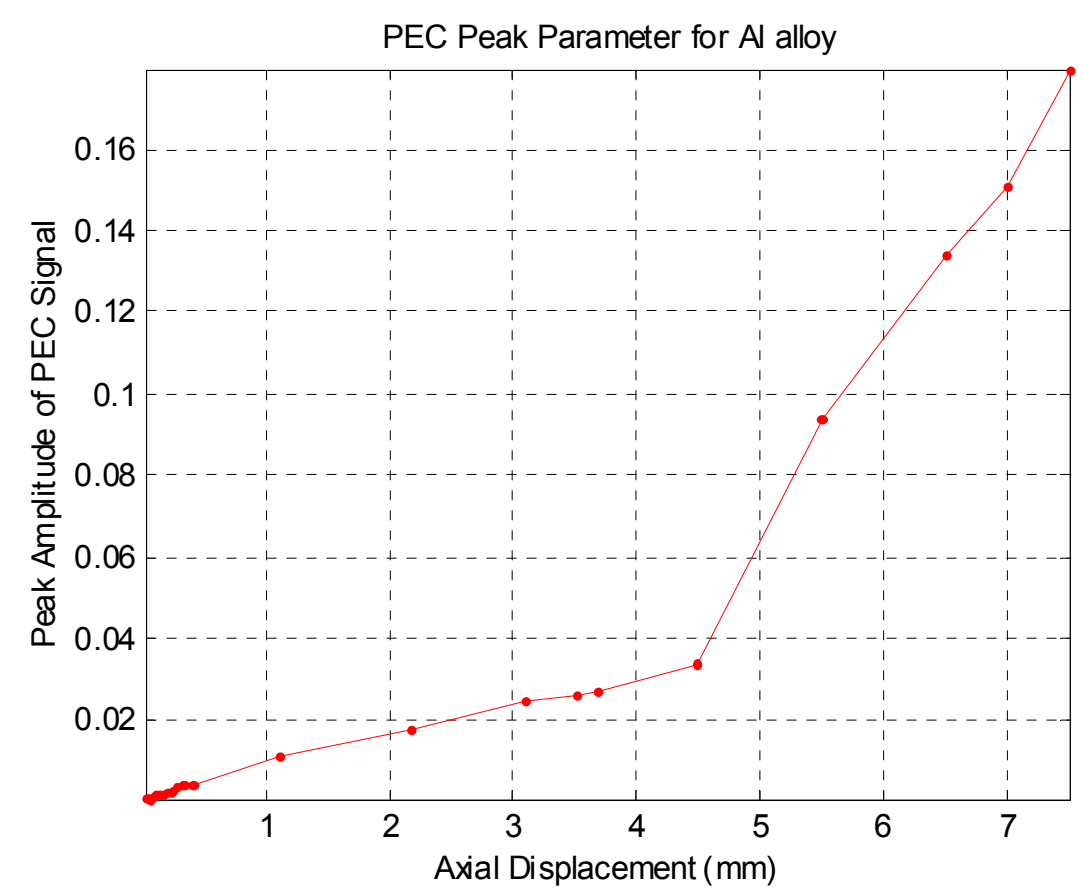

Fig. 3 PEC response to plastic deformation for Aluminum alloy sample during continuous loading. 


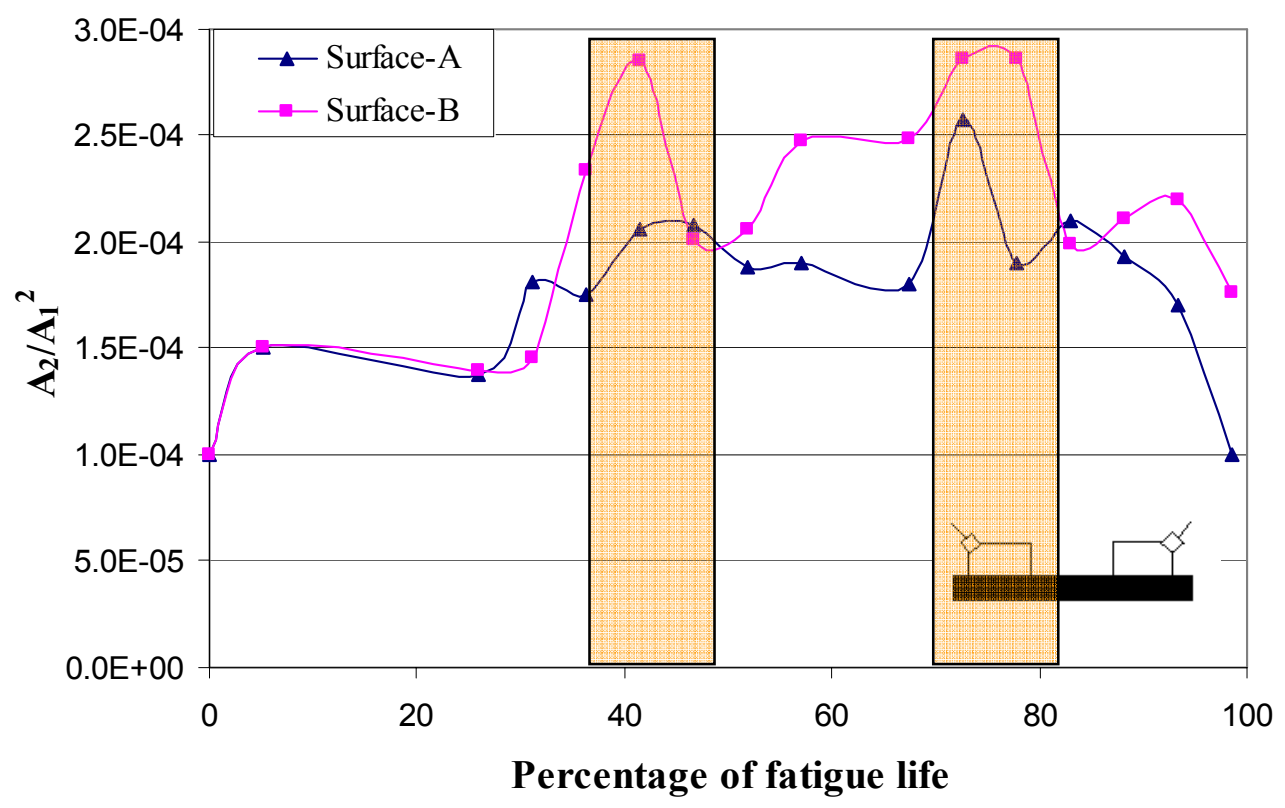

Fig. 4 Variation of nonlinear parameter $\left(\mathrm{A}_{2} / \mathrm{A}_{1}{ }^{2}\right)$ with percentage of fatigue life in $\mathrm{Al}$ alloy specimen.

\section{$\Delta \mathbf{T}_{\text {diss }} /$ cycle $=\Delta \tau_{\text {diss }}$}

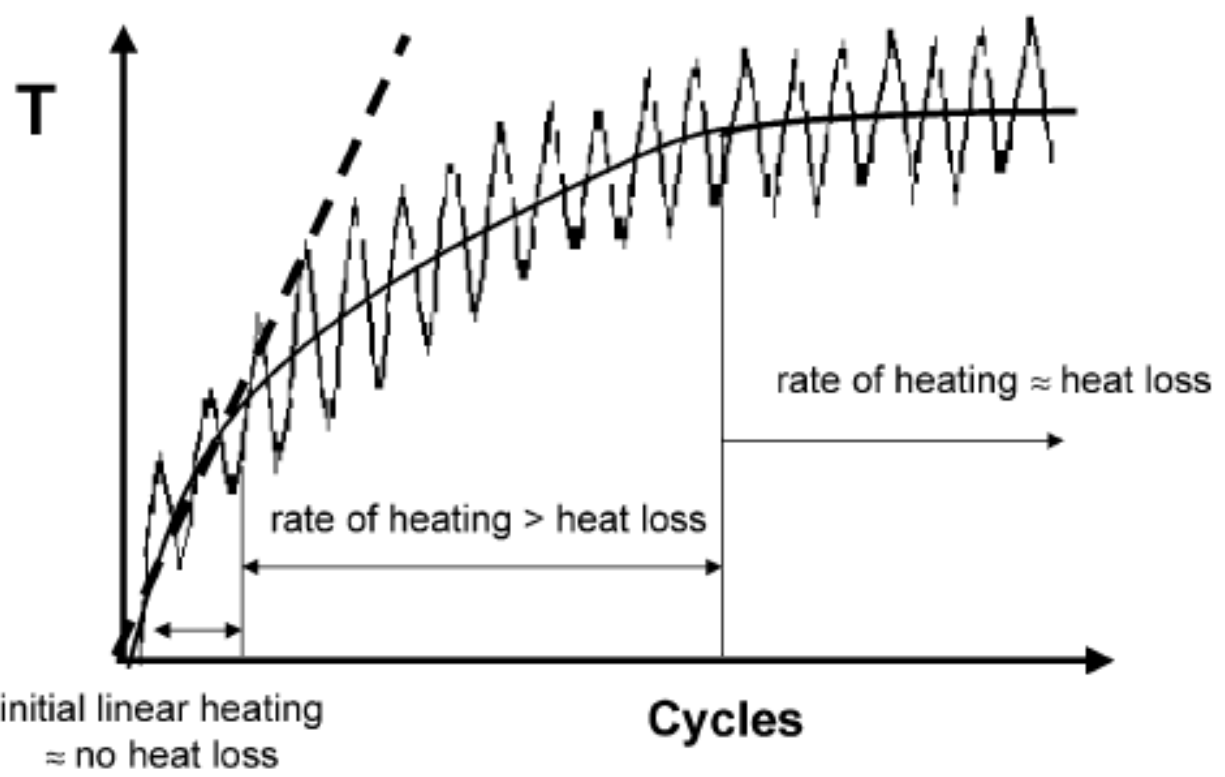

Fig. 5 Temperature change during cyclic loading shown schematically as a function of cycles. [12]. 


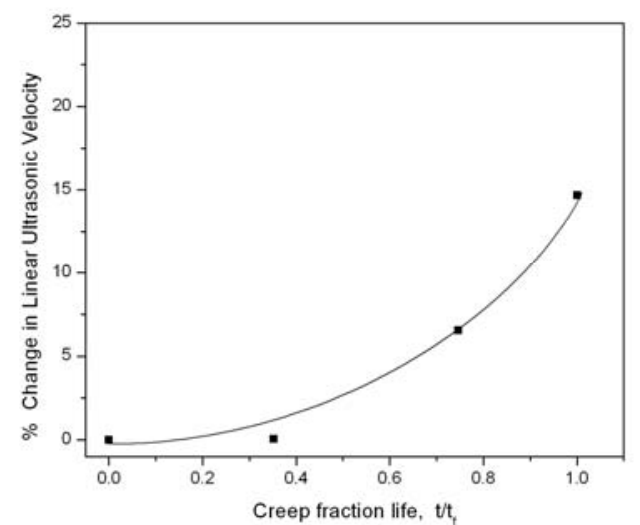

Fig. 6 Percentage change in linear ultrasonic velocity as a function of creep fraction life

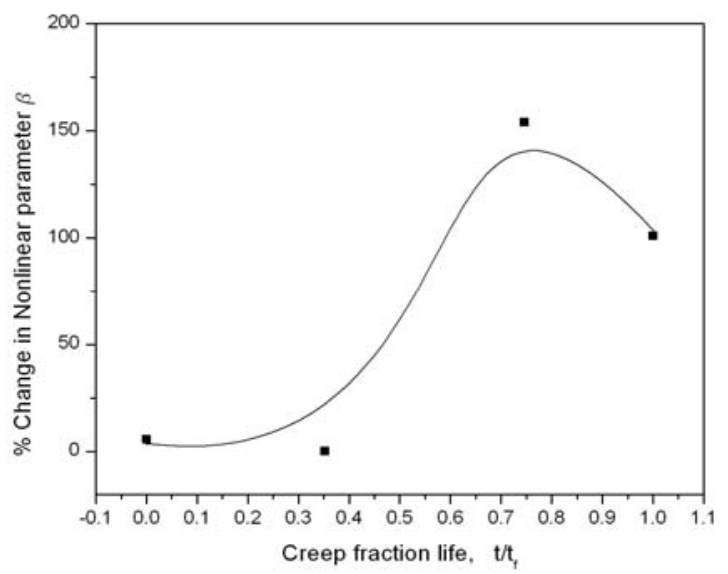

Fig 7. Variation of $\%$ change of Non-linear parameter with creep fraction life

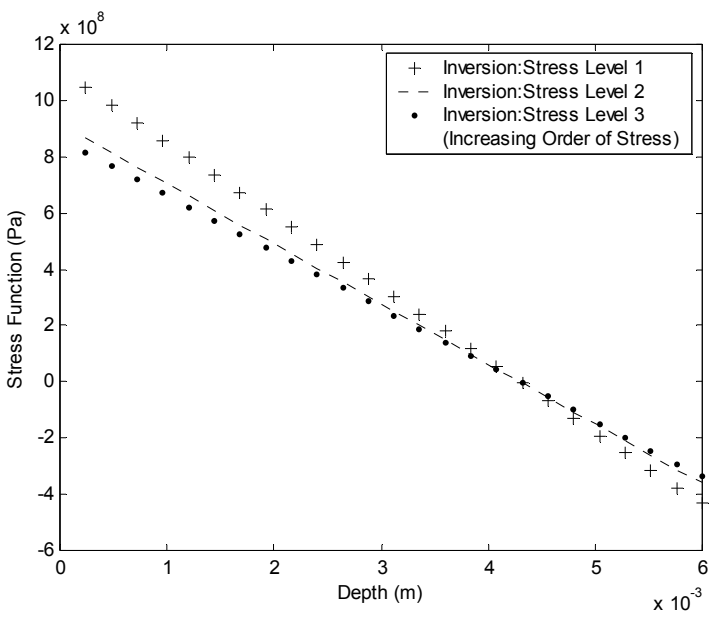

Fig 8 : The results obtained from the experiments on the 4-point bending test for a typical case of loading on AL sample. The Stress Measured vs. Depth is inverted using the inversion algorithm using Raleigh wave dispersion.

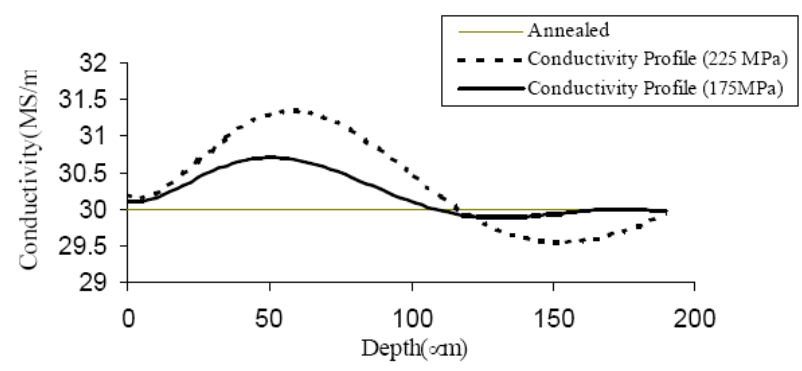

Fig. 9: Conductivity Profiles obtained from Multi-frequency Eddy Current Inverse model for samples that were peened at different water-jet pressures. 